\title{
An Absence of Radio-loud Active Galactic Nuclei in Geometrically Flat Quiescent Galaxies: Implications for Maintenance-mode Feedback Models
}

\author{
Ivana Barišić $^{1}$ (D), Arjen van der Wel ${ }^{1,2}$ (D), Josha van Houdt ${ }^{1}$, Michael V. Maseda ${ }^{3}$ (D), Eric F. Bell ${ }^{4}$ (D), Rachel Bezanson ${ }^{5}$ (D) \\ Yu-Yen Chang ${ }^{6}$ (D), Huub Röttgering ${ }^{7}$, Glenn van de Ven ${ }^{8}$ (D), and Po-Feng Wu ${ }^{1}$ (D) \\ ${ }^{1}$ Max-Planck Institut für Astronomie, Königstuhl 17, D-69117, Heidelberg, Germany; barisic@mpia.de \\ 2 Sterrenkundig Observatorium, Department of Physics and Astronomy, Ghent University, Belgium \\ ${ }^{3}$ Leiden Observatory,Leiden University, P.O. Box 9513, NL-2300 AA Leiden, The Netherlands \\ ${ }^{4}$ Department of Astronomy, University of Michigan, 1085 South University Avenue, Ann Arbor, MI 48109, USA \\ ${ }^{5}$ University of Pittsburgh, Department of Physics and Astronomy, 100 Allen Hall, 3941 OHara Street, Pittsburgh, PA 15260, USA \\ ${ }^{6}$ Academia Sinica Institute of Astronomy and Astrophysics, No.1 Section 4 Roosevelt Road, 11F of Astro-Math Building, Taipei 10617, Taiwan \\ ${ }^{7}$ Leiden Observatory, Leiden University, P.O. Box 9513, NL-2300 RA Leiden, The Netherlands \\ ${ }^{8}$ European Southern Observatory, Karl-Schwarzschild-Strae 2, D-85748 Garching bei Munchen, Germany \\ Received 2018 November 20; revised 2019 January 31; accepted 2019 January 31; published 2019 February 12
}

\begin{abstract}
Maintenance-mode feedback from low-accretion-rate active galactic nuclei (AGNs), manifesting itself observationally through radio-loudness, is invoked in all cosmological galaxy formation models as a mechanism that prevents excessive star formation in massive galaxies $\left(M_{*} \gtrsim 3 \times 10^{10} M_{\odot}\right)$. We demonstrate that at a fixed mass the incidence of radio-loud (RL) AGNs $\left(L>10^{23} \mathrm{~W} \mathrm{~Hz}^{-1}\right)$ identified in the Faint Images of the Radio Sky at Twenty centimeter and NRAO Very Large Array Sky Survey radio surveys among a large sample of quiescent (non-star-forming) galaxies selected from the Sloan Digital Sky Survey is much higher in geometrically round galaxies than in geometrically flat, disk-like galaxies. As found previously, the RL AGN fraction increases steeply with stellar velocity dispersion $\sigma_{*}$ and stellar mass, but even at a fixed velocity dispersion of $200-250 \mathrm{~km} \mathrm{~s}^{-1}$ this fraction increases from $0.3 \%$ for flat galaxies (projected axis ratio of $q<0.4$ ) to $5 \%$ for round galaxies $(q>0.8)$. We rule out the hypothesis that this strong trend is due to projection effects in the measured velocity dispersion. The large fraction of RL AGNs in massive, round galaxies is consistent with the hypothesis that such AGNs deposit energy into their hot gaseous halos, preventing cooling and star formation. However, the absence of such AGNs in disk-like quiescent galaxies - most of which are not satellites in massive clusters, raises important questions. Is maintenance-mode feedback a generally valid explanation for quiescence? If so, how does that feedback avoid manifesting at least occasionally as an RL galaxy?
\end{abstract}

Key words: galaxies: active - galaxies: fundamental parameters - galaxies: star formation - galaxies: statistics galaxies: structure

\section{Introduction}

To explain the mismatch between the theoretical dark matter halo mass function and the observed galaxy stellar mass function, semi-analytical and hydrodynamical simulations of galaxy formation and evolution have implemented a set of feedback mechanisms to reduce the efficiency of star formation; they rely on supernova feedback in low halo mass systems, and supermassive black hole (SMBH) feedback in systems with high-mass halos (Bower et al. 2006; Croton et al. 2006; Fabian 2012; Vogelsberger et al. 2014; Pillepich et al. 2018). The most common picture states that the SMBH feedback generally manifests through radio-loudness in active galactic nuclei (AGNs) in the form of the jets ("radio-mode") or gas outflows ("quasar-mode") as a consequence of a low or high accretion rate (respectively) of the gas onto the SMBH. This feedback picture states that powerful jets transfer their energy to the surrounding gas, heating it up and therefore preventing it from cooling and forming new stars. "Radiomode" feedback has thus been introduced as the leading mechanism in explaining the mass growth deficiency in the most massive early-type galaxies with SMBH.

Direct observational evidence for this picture in local galaxies comes from observations of cavities in the X-rayemitting gas caused by jets, connecting the presence of radioloud (RL) AGNs with the absence of star formation
(McNamara \& Nulsen 2007; Heckman \& Best 2014). Indirect evidence in the local universe has also provided additional support to this radio-mode feedback picture (Matthews et al. 1964; Kauffmann et al. 2003), including studies that show a strong, increasing trend in the RL fraction with $M_{*}$ and stellar velocity dispersion $\sigma_{*}$ (Best et al. 2005). According to the van den Bosch (2016) black hole (BH) mass $-\sigma_{*}$ relation (see also Gebhardt et al. 2003; Beifiori et al. 2012), this implies an increasing trend of the RL fraction with the $\mathrm{BH}$ mass, suggesting the importance of the $\mathrm{BH}$ mass as a parameter in setting the probability for a galaxy to become RL (see also Häring \& Rix 2004; Terrazas et al. 2017).

In this Letter we explore how the incidence rate of RL AGN varies with geometric shape (flat or disk-like versus round). We test this by examining the correlation of the RL fraction with $\sigma_{*}$ and the projected axis ratio for a sample of galaxies drawn from the Sloan Digital Sky Survey (SDSS; York et al. 2000), NRAO Very Large Array Sky Survey (NVSS; Condon et al. 1998) and Faint Images of the Radio Sky at Twenty centimeter (FIRST; Becker et al. 1995) survey, and we present evidence that round galaxies host RL AGN much more frequently than disk-like galaxies at any fixed $\sigma_{*}$ and $M_{*}$. According to the "radio-mode" feedback picture, and the association of the presence of RL AGN with quiescence, this raises the question of why these galaxies are not forming new stars in the absence of a visible heating source. 
In Section 2 we give an overview of the data set that we use in this study, and in Section 3 we present or results and discuss the implications. In Section 4 we give a brief summary of our main results. We use standard cosmology: $H_{0}=70\left(\mathrm{~km} \mathrm{~s}^{-1}\right) \mathrm{Mpc}^{-1}$, $\Omega_{M}=0.3, \Omega_{\Lambda}=0.7$

\section{Data and Sample Selection}

\subsection{SDSS}

The SDSS survey (York et al. 2000) provides unique and high-quality information on various physical properties of a large number of present-day galaxies. Physical properties of those galaxies are measured and derived using photometry and spectroscopy, and include $\sigma_{*},{ }^{9}$ star formation rate (SFR), $M_{*}$, redshift, and projected axis ratio. The resolution of the spectroscopic data set is $R=1850-2200$, covering the wavelength range between 3800 and $9200 \AA$, with a minimum signal-to-noise ratio $(\mathrm{S} / \mathrm{N}) \mathrm{S} / \mathrm{N} \sim 14 \AA^{-1}$.

In this Letter we make use of the SFRs and $M_{*}$ obtained from modeling galaxy spectral energy distributions (SEDs; Chang et al. 2015) based on SDSS Data Release 7 (DR7; Abazajian et al. 2009) and the Wide-field Infrared Survey Explorer (Cutri et al. 2013).

In order to create a volume-selected sample $\left(303,785^{10}\right)$ complete in $M_{*}$ we apply a redshift-dependent mass-cut: $\log \left(M_{*}\right)>10.6+2.28 \times \log (\mathrm{z} / 0.1) \quad($ Chang et al. 2015) consequently spanning a redshift range from $0.02<z<$ 0.14 . For sample classification between star-forming and quiescent galaxies, we apply a color-based criteria following Chang et al. $(2015 ; u-r>1.6 \times(r-z)+1.1)$ for quiescent galaxies. Star-forming galaxies are considered to be all other galaxies that fall outside of this color-based criteria.

\subsection{NVSS and FIRST}

The NVSS (Condon et al. 1998) $1.4 \mathrm{GHz}$ sky survey north of $\delta=-40^{\circ}$ was conducted between 1993 and 1996. The angular resolution of the observed data set is $45^{\prime \prime}$, with the noise level rms of $0.45 \mathrm{mJy}^{\text {beam }}{ }^{-1}$ and the point-source flux density $50 \%$ completeness level at $2.5 \mathrm{mJy}$. For more details see Condon et al. (1998). The FIRST (Becker et al. 1995) $1.4 \mathrm{GHz}$ survey of the northern Galactic cap was conducted between 1993 and 2004. The average rms of the images is $0.15 \mathrm{mJy}$, with an angular resolution of $5^{\prime \prime}$ and the flux density limit of $1 \mathrm{mJy}$. Both surveys have been conducted using the Very Large Array (VLA) radio interferometer.

Taking advantage of the high-resolution FIRST data set, Best \& Heckman (2012) cross-matched sources with galaxies in the SDSS DR7. Flux density measurements are subsequently performed on the NVSS data set. The resulting cross-matched catalog, with a limiting NVSS flux density value of $5 \mathrm{mJy}$, is complete for RL AGN down to $L_{1.4 \mathrm{GHz}} \sim 10^{23} \mathrm{~W} \mathrm{~Hz}^{-1}$ at $z=0.1$. Because the sample is incomplete below $L_{1.4 \mathrm{GHz}}$ $<2.5 \times 10^{23} \mathrm{~W} \mathrm{~Hz}^{-1}$ at highest redshifts, we apply a volume-correction that reaches at most a factor 4 for $L_{1.4 \mathrm{GHz}}$ $\sim 10^{23} \mathrm{~W} \mathrm{~Hz}^{-1}$ at $z=0.14$.

\footnotetext{
9 http://classic.sdss.org/dr7/algorithms/veldisp.html

${ }^{10}$ We removed 11,058 galaxies (3.5\%) that lack rest-frame $u$-, $r$-, and $z$-band photometry and a measurement of the axis ratio.
}

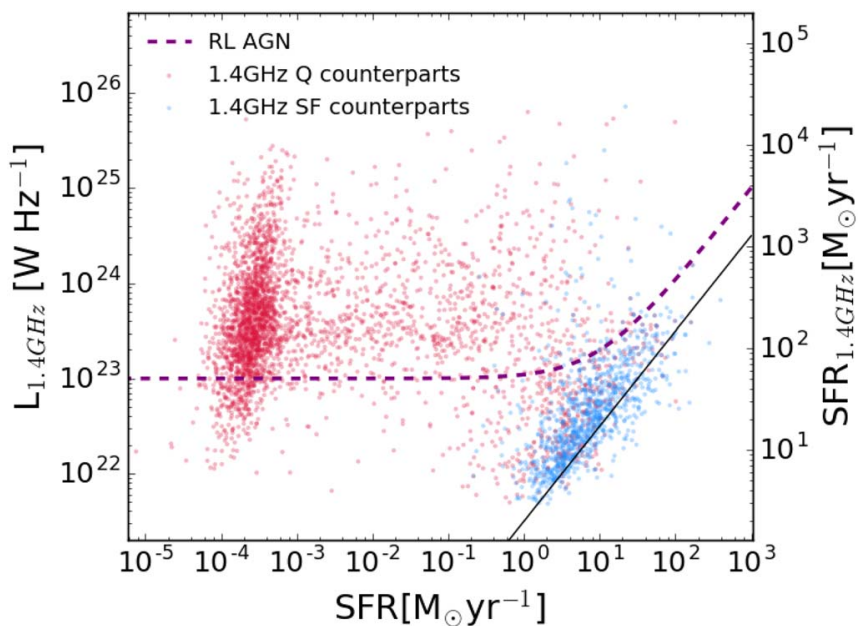

Figure 1. 1.4 GHz luminosity vs. mid-infrared (mid-IR) SFR estimates (Chang et al. 2015) for quiescent (red) and star-forming (blue) galaxies. Right-handside $y$-axis converts the $1.4 \mathrm{GHz}$ luminosity into SFR. All data points have a radio counterpart in the NVSS, and the purple line indicates selected RL AGNs. The selection reflects the minimum required AGN luminosity of $10^{23} \mathrm{~W} \mathrm{~Hz}^{-1}$ and accounts for a contribution of star formation (see the text for details). The clump of galaxies at low SFR reflects the minimum SFR allowed in the SED model used by Chang et al. (2013) and has no physical meaning.

\subsection{Selection of RL AGN Hosts}

To select an RL sub-sample we consider galaxies with detected radio emission at $1.4 \mathrm{GHz}$ by the NVSS and FIRST. From the total number of galaxies in the selected galaxy sample, the number of those detected with $F_{1.4 \mathrm{GHz}}>5 \mathrm{mJy}$ is 4571 , and they are shown with red and blue symbols in Figure 1.

We use Condon (1992) luminosity relation $(\alpha=-0.8)$, to convert the NVSS measured flux density values $F_{1.4 \mathrm{GHz}}$ into luminosities. Derived luminosities at $1.4 \mathrm{GHz}$ are then converted to radio-based SFRs using the Bell (2003) radiofar-infrared (far-IR) calibration.

We follow the Best et al. (2005) $1.4 \mathrm{GHz}$ luminosity limit $L_{1.4 \mathrm{GHz}}>10^{23} \mathrm{~W} \mathrm{~Hz}^{-1}$ to select RL galaxies. We subtract the contribution of star formation as follows: $L_{1.4 \mathrm{GHz}}-$ $\left(3 \times L_{1.4 \mathrm{GHz}, \mathrm{SFR}}\right)>10^{23}$, where $L_{1.4 \mathrm{GHz}}$ is the luminosity based on the $1.4 \mathrm{GHz}$ flux density value, and $L_{1.4 \mathrm{GHz} \text {,SFR }}$ is the expected $L_{1.4 \mathrm{GHz}}$ luminosity based on the SFR (taken from Chang et al. 2015). In order to select RL AGN with high confidence we multiply $L_{1.4 \mathrm{GHz}, \mathrm{SF}}$ by a factor 3 to account for uncertainties in radio- and mid-IR based SFR estimates.

We keep the color-based classification from Chang et al. (2015) to separate the radio-detected sample between quiescent (red) and star-forming (blue) galaxies. The selected RL AGN sub-sample is indicated with the purple dashed line in Figure 1.

\section{Which Parameters Correlate the RL AGN Fraction?}

In the following subsections we explore the RL fraction trend with the $\sigma_{*}$ (or equivalently $\mathrm{BH}$ mass; van den Bosch 2016) for all, quiescent, and star-forming galaxies. We also explore the aspect of radio-loudness occurring more frequently among galaxies of a specific shape.

\subsection{RL AGN Fraction Increases with $\sigma_{*}$}

It has been shown in the local universe that the RL fraction of massive elliptical galaxies exhibits a strong increasing trend 

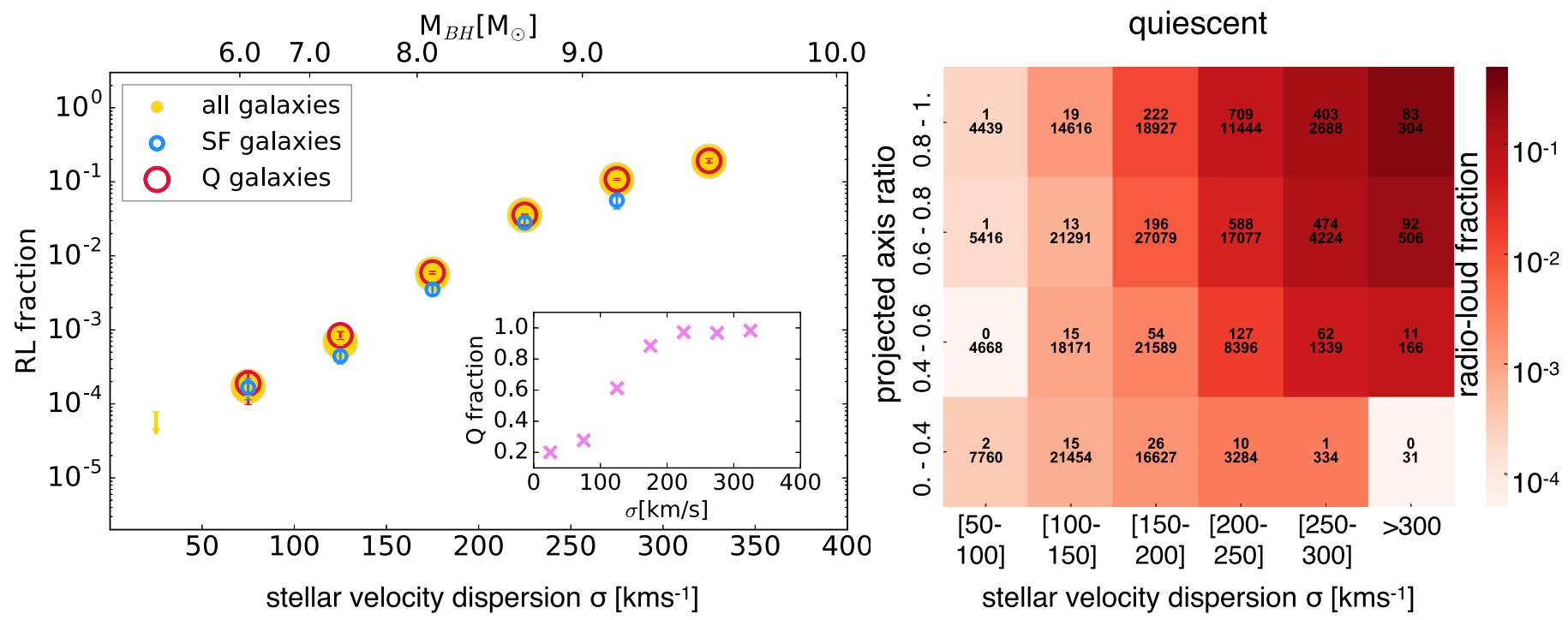

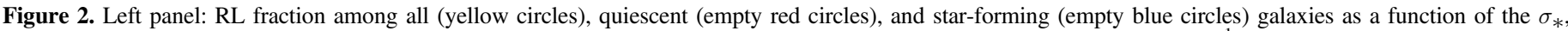

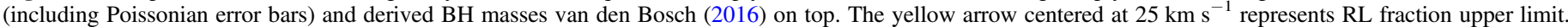

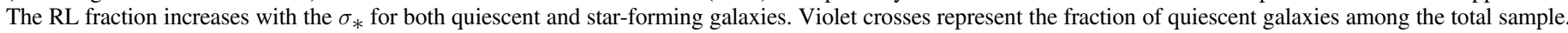

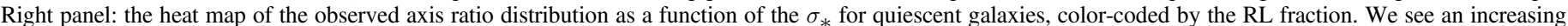
trend in the RL fraction with $\sigma_{*}$ and with the projected axis ratio.

with $\sigma_{*}$ (Best et al. 2005). According to the BH mass $-\sigma_{*}$ relation (van den Bosch 2016) this implies a strong dependence of the RL fraction on the BH mass. In Figure 2 (left panel) we show the fraction of quiescent galaxies in the selected sample (violet crosses), and the RL fraction among all (yellow circles), quiescent (empty red circles), and star-forming (empty blue circles) galaxies as a function of $\sigma_{*}$ and the corresponding $\mathrm{BH}$ mass (van den Bosch 2016). It is evident that the RL fraction exhibits an increasing trend with $\sigma_{*}$, which reproduces the result from Best et al. (2005).

Evidently, the RL fraction is increasing among star-forming galaxies as well, and these galaxies appear to host RL AGN as frequently as quiescent galaxies at fixed $\sigma_{*}$ (i.e., Janssen et al. 2012). Because the star-forming galaxies host RL AGNs at a comparable rate as the quiescent galaxies, then the radioloudness is not a sufficient condition for a galaxy to become quiescent.

\subsection{Geometry Plays a Role in RL Fraction}

For the remainder of this Letter we only consider quiescent galaxies, as we are interested in constraining maintenancemode feedback. We divide the sample into six evenly spaced bins of $\sigma_{*}$ and four in the projected axis ratio. In each bin we determine the fraction of RL galaxies. The right panel of Figure 2 presents the heat map of the projected axis ratio distribution as a function of $\sigma_{*}$ for quiescent galaxies, colorcoded by the fraction of RL galaxies. The top number inside of each bin corresponds to the number of RL galaxies, while the bottom number corresponds to the total number of galaxies. We observe that the RL fraction among quiescent galaxies exhibits an increasing trend with both parameters $-\sigma_{*}$ and the projected axis ratio.
This result illustrates that the important parameters in determining the probability for a galaxy to "switch on" as an RL AGN is not only its $\sigma_{*}$ but also the geometry of the galaxy.

We note that the $\sigma_{*}$ measurement for all but face-on galaxies is to a certain degree affected by a contribution from the rotational velocity. Due to this effect, the actual $\sigma_{*}$ should be lower for inclined galaxies, further suggesting lower RL fractions. Assuming that the $M_{*}$ roughly corresponds to the virial mass, then the ratio between the galaxy $M_{*}$ and its inclination-independent semimajor axis (half-light) radius $R$, can serve as a proxy for the intrinsic $\sigma_{*}$ free of the rotational velocity effects, and independent on the inclination. Figure 3 shows the RL fraction trend with the projected axis ratio for quiescent galaxies, in narrow bins of the $\sigma_{*}$ (left panel), $M_{*}$ (middle panel), and the $\sigma_{*}$ proxy $\log \left(M_{*} / R\right)$ (right panel). These trends are representative of the sample as a whole. The increasing trend in all three panels is likely dominated by a dependence of the RL fraction on the intrinsic shape of the galaxy, rather then its inclination. We note that replacing the semimajor axis radius with the inclination-independent circularized radius in estimating the $\sigma_{*}$ proxy $\log \left(M_{*} / R\right)$ results in an even stronger trend than seen in the right panel of Figure 3. Therefore, correcting the $\sigma_{*}$ measurement of inclined galaxies for the rotational velocity effects would not significantly alter the increasing trend we see, which implies the preference for RL AGN to occur in round galaxies.

\subsection{RL AGN Fraction of Intrinsically Round and Flat Galaxies}

Chang et al. (2013) developed a two-population model to reproduce the observed axis ratio distribution of quiescent galaxies up to $z=2.5$, combining round (triaxial) and flat (oblate) populations of galaxies. They perform their modeling in three different mass bins, and to simplify the analysis we only use the fit parameters of the highest stellar mass bin 

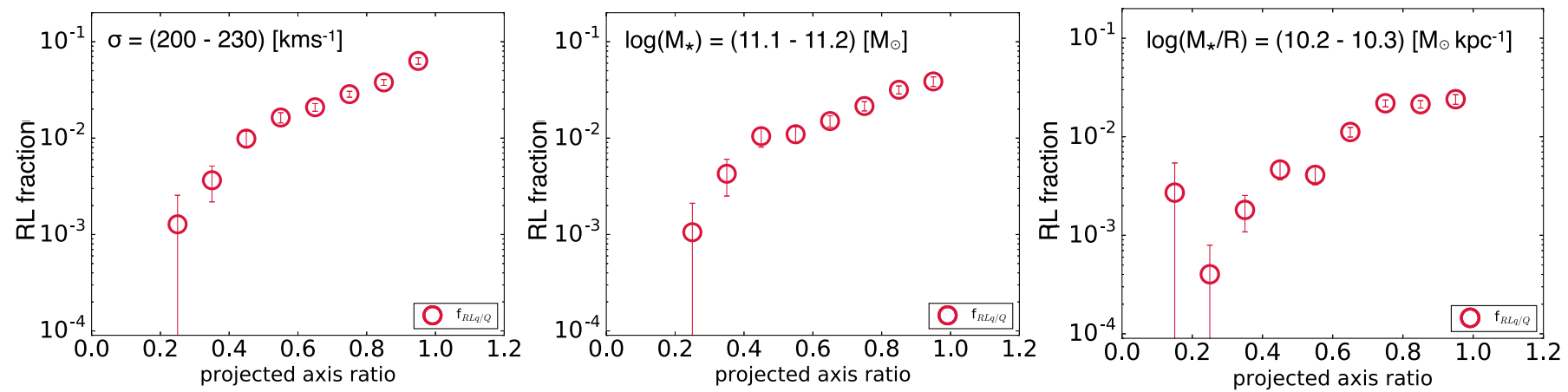

Figure 3. RL fraction among quiescent galaxies as a function of the projected axis ratio in the narrow bins of the $\sigma_{*}\left(\right.$ left panel), $M_{*}$ (middle panel), and $M_{*} / R$ as a $\sigma_{*}^{2}$ proxy (right panel). RL fraction exhibits an increasing trend with the observed axis ratio in all three panels.
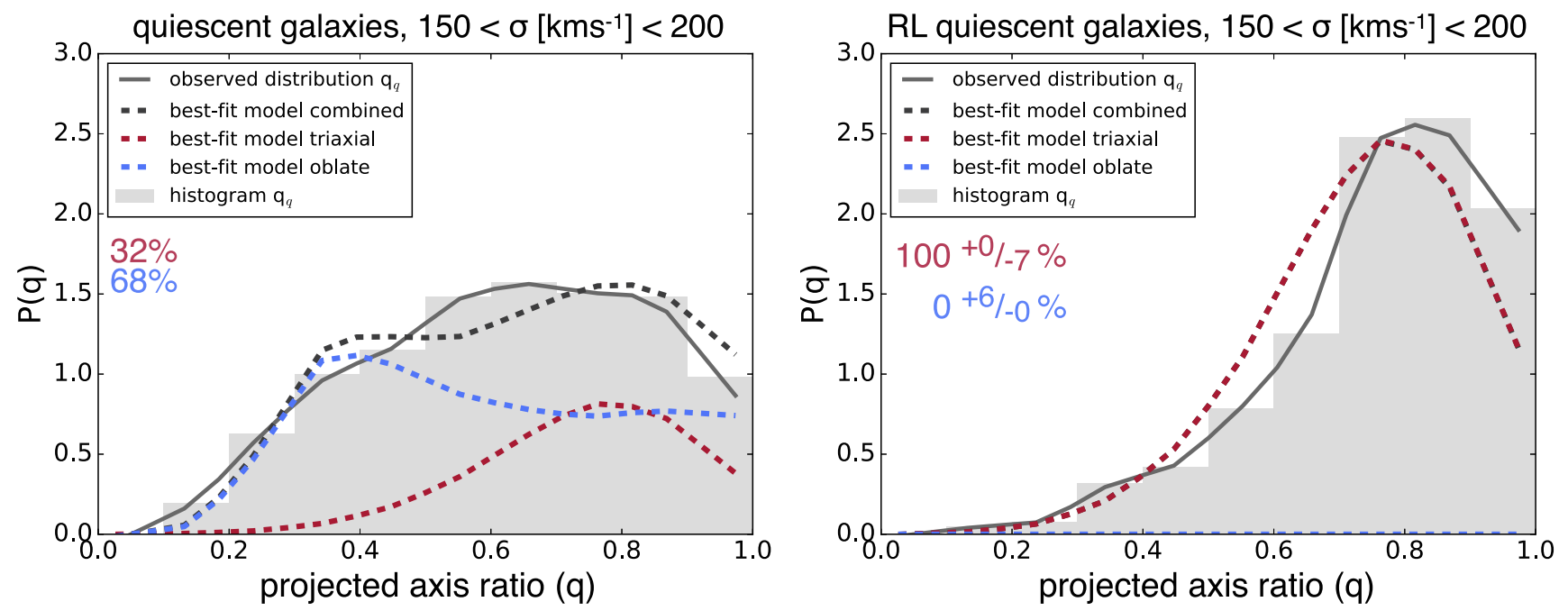

Figure 4. Normalized histogram of the observed axis ratio distribution (gray bins) for quiescent (left panel) and RL-quiescent (right panel) galaxies and the function describing the observed axis ratio distribution (gray curve) in the $\sigma_{*}$ bin $150-200 \mathrm{~km} \mathrm{~s}^{-1}$. We also show the best-fit two-component model probability distribution (black dashed curve), and the underlying triaxial (red dashed curve) and oblate (blue dashed curve) best-fit fractions.

$\left(M_{*}>10.8\right),{ }^{11}$ where the intrinsic axis ratio of the oblate component is $b^{12}=0.29 \pm 0.07$. The best-fit triaxility $\left(T^{13}=\right.$ $0.64 \pm 0.06)$ and ellipticity $\left(E^{14}=0.41 \pm 0.02\right)$ parameter values, and their standard deviations $\left(\sigma_{T}, \sigma_{E}=0.08 \pm 0.05\right.$, $0.19 \pm 0.02)$ are used to generate distributions of intrinsic intermediate-to-long axis ratio $\beta$ and short-to-long axis ratio $\gamma$. Combining these distributions with random viewing angles provides us with model triaxial and oblate intrinsic axis ratio distributions.

We split the sample of quiescent galaxies into six equally spaced $\sigma_{*}$ bins, and in each bin we find the best-fit linear combination of model triaxial and oblate shape distributions that reproduce the observed axis ratio distribution. This gives us the triaxial and oblate fraction of galaxies in the specific $\sigma_{*}$ bin. As an example, in Figure 4 we show a normalized histogram (gray bins) of the observed axis ratio $(q)$ distribution, and a corresponding function describing the distribution obtained after binning, for quiescent (left panel) and for RLquiescent (right panel) galaxies in the range of $\sigma_{*}$ values from 150 to $200 \mathrm{~km} \mathrm{~s}^{-1}$. For illustration, we choose the $\sigma_{*}$ bin in

\footnotetext{
${ }^{11}$ This mass limit corresponds to a $\sigma_{*}$ limit of $\sim 120 \mathrm{~km} \mathrm{~s}^{-1}$, but even below this limit the shape parameters are not very different and our analysis would not be affected.

${ }^{12} b=$ intermediate axis of the triaxial ellipsoid.

${ }^{13} T=\left[1-\beta^{2}\right] /\left[1-\gamma^{2}\right]$.

${ }^{14} E=1-\gamma$.
}

which the total population is similarly populated by both triaxial and oblate galaxies according to the two-population model. Blue and red dashed curves represent best-fit oblate and triaxial fractions, respectively. We see that the triaxial fraction $\left(100 \pm{ }_{7}^{0} \%\right)$ of RL AGN in the observed quiescent population in this $\sigma_{*}$ bin completely dominates over the oblate fraction, implying a more frequent occurrence of RL AGN among round galaxies.

The best-fit model triaxial and oblate fraction for the observed axis ratio distribution of quiescent galaxies can be rescaled to fit the observed axis ratio distribution of RLquiescent galaxies. Multiplying these rescaling factors by the $\mathrm{RL}$ fraction gives the RL fraction among triaxial and oblate galaxies.

Figure 5 shows the RL fraction among triaxial (filled red squares) and oblate (open red squares) galaxies for quiescent population as a function of the $\sigma_{*}$. The overall triaxial fraction (black crosses) increases with the $\sigma_{*}$. The increase in RL fraction with $\sigma_{*}$ is entirely due to its increase among the triaxial population. In fact, the inferred RL fraction among oblate population is essentially zero. This analysis, by accounting for round (face-on) disk-like galaxies, further strengthens our result that RL AGNs preferentially reside in intrinsically round galaxies. We note that we do not claim that the RL fraction among disk-like quiescent galaxies is, in fact, zero; the triaxial population consists of objects with a wide 


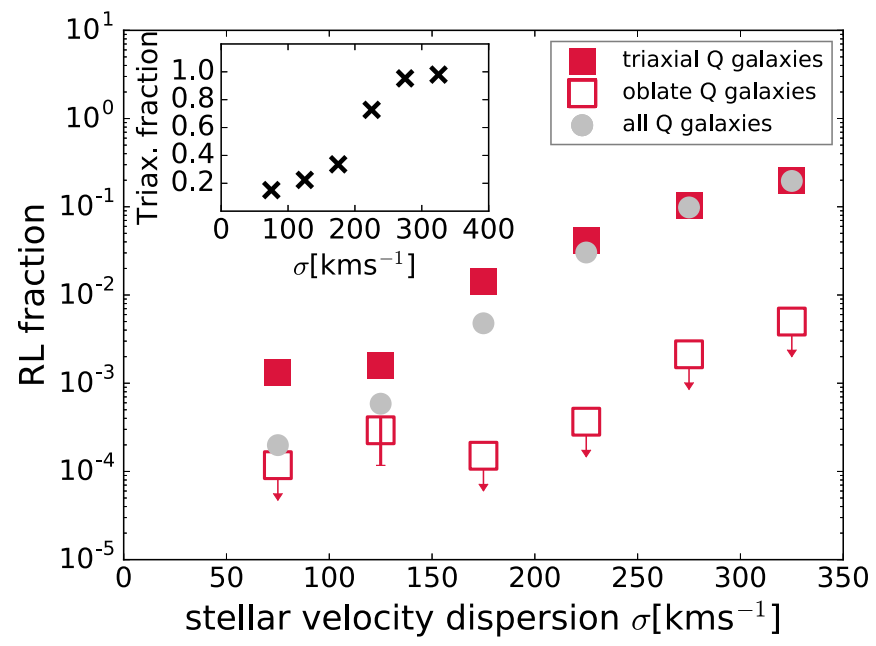

Figure 5. RL fraction among triaxial (filled red squares) and oblate (open red squares) galaxies as a function of the $\sigma_{*}$ for quiescent galaxies. The RL fraction is higher among triaxial galaxies. The plot also shows the RL fraction among quiescent galaxies (gray circles), and the best-fit triaxial fraction (black crosses).

range in intrinsic $\beta$ and $\gamma$ values (see above), including a subset with $\beta \sim 1$ and small $\gamma$, that is, disk-like geometries.

\subsection{Satellites, Centrals, and Halo Mass}

We now investigate whether or not the correlation between the shape and RL fraction is driven by an underlying connection between dark matter halo properties and RL AGN. This may be expected in the case that disk-like galaxies live in less-massive halos than round galaxies, or if satellite galaxies, which are more often disk-like than centrals (van der Wel et al. 2010), are less likely to be RL AGN.

We cross-match our sample with the SDSS DR7-based "petroC" halo catalog, centrals are chosen based on stellar mass, and halo masses are assigned to groups based on their total stellar mass. We focus on quiescent galaxies with $200<$ $\sigma_{*} /\left(\mathrm{km} \mathrm{s}^{-1}\right)<230$, which shows a strong correlation between the shape and RL AGN incidence, and for which the satellite fraction is non-negligible ( $\sim 20 \%$, van den Bosch et al. 2008). We find that RL fractions are similar: $2.3 \%$ for the satellites and $2.8 \%$ for the centrals, and their trends with galaxy shape are consistent with each other. There is no substantial difference $(<0.15$ dex $)$ between the halo masses of round central galaxies and flat central galaxies, and selecting galaxies by halo mass still contains a strong correlation between RL AGN fraction and galaxy shape.

We conclude that the strong dependence of RL AGN incidence on galaxy shape is intrinsic and cannot be explained by differences between RL AGN fractions in satellite and central galaxies, nor to differences in halo mass.

\section{Discussion}

In this Letter we demonstrate that RL AGNs occur more frequently in round than in flat quiescent galaxies at any $M_{*}$ or $\sigma_{*}$. The analysis of the projected axis ratio distribution of RL AGNs hosted by quiescent galaxies shows that the increased $\mathrm{RL}$ fraction at higher $\sigma_{*}$ values is entirely driven by

\footnotetext{
15 http://gax.sjtu.edu.cn/data/Group.html
}

intrinsically round (triaxial) galaxies. The RL fraction reaches $\sim 20 \%$ for the most massive, round quiescent galaxies $\left(\sigma_{*} \sim 300 \mathrm{~km} \mathrm{~s}^{-1}\right)$, whereas geometrically flat (disk-like) quiescent galaxies have an extremely low RL AGN fraction $\left(<\sim 10^{-3}\right)$, even at high $\sigma_{*}\left(>200 \mathrm{~km} \mathrm{~s}^{-1}\right)$.

This result is consistent with the common notion that luminous radio AGN reside in massive, elliptical galaxies. However, thus far, the correlation between the RL AGN occurrence in quiescent galaxies and a second global property, in addition to its $\sigma_{*}(\mathrm{BH}$ mass), has not been explicitly demonstrated. The roundness of a galaxy likely reflects its merger history, and the connection between an active merger history and the occurrence of lowaccretion rate radio AGNs has been made by many authors (De Ruiter et al. 2005; Capetti \& Balmaverde 2006; Sikora et al. 2007; Fanidakis et al. 2010). The physical basis for this connection has been referred to as the "spin paradigm" (Blandford \& Znajek 1977; Wilson \& Colbert 1995; Hughes \& Blandford 2003; McKinney \& Gammie 2004), in which only BHs with large spin parameters can launch radio jets. In turn, a large spin parameter is the result of $\mathrm{BH}-\mathrm{BH}$ mergers that reflect the merger history of the host galaxy.

Regardless of the physical explanation, the lack of RL AGN in disk-like quiescent galaxies raises important questions. In galaxy formation and evolution models, low-accretion rate AGNs are universally assumed to play a crucial role in preventing excessive star formation in massive galaxies, independent of their structure. Our findings suggest that, if this model is generally valid, the AGN feedback does not manifest itself at radio wavelengths for galaxies with disk-like structures. We conclude that there is compelling evidence for maintenance-mode feedback for intrinsically round galaxies, but that further study is needed to bolster the evidence for such feedback as a general explanation for the lack of star formation activity in massive galaxies.

We thank the anonymous referee for providing useful feedback. Funding for the SDSS and SDSS-II has been provided by the Alfred P. Sloan Foundation, the Participating Institutions, the National Science Foundation, the U.S. Department of Energy, the National Aeronautics and Space Administration, the Japanese Monbukagakusho, the Max Planck Society, and the Higher Education Funding Council for England. ${ }^{16}$ This project has received funding from the European Research Council (ERC) under the European Union's Horizon 2020 research and innovation programme (grant agreement 683184). G.v.d.V. acknowledges funding from the European Research Council (ERC) under the European Union's Horizon 2020 research and innovation programme under grant agreement No. 724857 (Consolidator Grant ArcheoDyn).

\section{ORCID iDs}

Ivana Barišić (iD https://orcid.org/0000-0001-6371-6274 Arjen van der Wel (i) https://orcid.org/0000-0002-5027-0135 Michael V. Maseda (i) https://orcid.org/0000-0003-0695-4414 Eric F. Bell (i) https://orcid.org/0000-0002-5564-9873 Rachel Bezanson (iD https://orcid.org/0000-0001-5063-8254 Yu-Yen Chang (ib https://orcid.org/0000-0002-6720-8047 Glenn van de Ven (1) https://orcid.org/0000-0003-4546-7731 Po-Feng Wu (1) https://orcid.org/0000-0002-9665-0440

\footnotetext{
${ }^{16}$ http://www.sdss.org/
} 


\section{References}

Abazajian, K. N., Adelman-McCarthy, J. K., Agüeros, M. A., et al. 2009, ApJS, 182, 543

Becker, R. H., White, R. L., \& Helfand, D. J. 1995, ApJ, 450, 559

Beifiori, A., Courteau, S., Corsini, E. M., \& Zhu, Y. 2012, MNRAS, 419, 2497

Bell, E. F. 2003, ApJ, 586, 794

Best, P., \& Heckman, T. 2012, MNRAS, 421, 1569

Best, P. N., Kauffmann, G., Heckman, T. M., et al. 2005, MNRAS, 362, 25

Blandford, R. D., \& Znajek, R. L. 1977, MNRAS, 179, 433

Bower, R. G., Benson, A. J., Malbon, R., et al. 2006, MNRAS, 370, 645

Capetti, A., \& Balmaverde, B. 2006, A\&A, 453, 27

Chang, Y.-Y., van der Wel, A., da Cunha, E., \& Rix, H.-W. 2015, ApJS, 219, 8

Chang, Y.-Y., van der Wel, A., Rix, H.-W., et al. 2013, ApJ, 773, 149

Condon, J. 1992, ARA\&A, 30, 575

Condon, J. J., Cotton, W. D., Greisen, E. W., et al. 1998, AJ, 115, 1693

Croton, D. J., Springel, V., White, S. D. M., et al. 2006, MNRAS, 365, 11

Cutri, R. M., Wright, E. L., Conrow, T., et al. 2013, yCat, 2328, 1

De Ruiter, H. R., Parma, P., Capetti, A., et al. 2005, A\&A, 439, 487

Fabian, A. 2012, ARA\&A, 50, 455

Fanidakis, N., Baugh, C. M., Benson, A. J., et al. 2010, MNRAS, 410, 53

Gebhardt, K., Faber, S. M., Koo, D. C., et al. 2003, ApJ, 583, 92
Häring, N., \& Rix, H.-W. 2004, ApJL, 604, L89

Heckman, T. M., \& Best, P. N. 2014, ARA\&A, 52, 589

Hughes, S. A., \& Blandford, R. D. 2003, ApJL, 585, L101

Janssen, R. M. J., Röttgering, H. J. A., Best, P. N., \& Brinchmann, J. 2012, A\&A, 541, A62

Kauffmann, G., Heckman, T. M., Tremonti, C., et al. 2003, MNRAS, 346,1055

Matthews, T. A., Morgan, W. W., \& Schmidt, M. 1964, ApJ, 140, 35

McKinney, J. C., \& Gammie, C. F. 2004, ApJ, 611, 977

McNamara, B., \& Nulsen, P. 2007, ARA\&A, 45, 117

Pillepich, A., Springel, V., Nelson, D., et al. 2018, MNRAS, 473, 4077

Sikora, M., Stawarz, Ł., \& Lasota, J.-P. 2007, ApJ, 658, 815

Terrazas, B. A., Bell, E. F., Woo, J., \& Henriques, B. M. B. 2017, ApJ, 844,170

van den Bosch, F. C., Aquino, D., Yang, X., et al. 2008, MNRAS, 387, 79

van den Bosch, R. C. 2016, ApJ, 831, 134

van der Wel, A., Bell, E. F., Holden, B. P., Skibba, R. A., \& Rix, H.-W. 2010, ApJ, 714, 1779

Vogelsberger, M., Genel, S., Springel, V., et al. 2014, MNRAS, 444, 1518

Wilson, A., \& Colbert, E. 1995, BAAS, 27, 830

Yang, X., Mo, H. J., van den Bosch, F. C., et al. 2007, ApJ, 671, 153

York, D. G., Adelman, J., Anderson, J. E. Jr., et al. 2000, AJ, 120, 1579 